\title{
An Investigation of Retirement Expectation among Medical Technologists in Korea
}

\author{
Yoon-kyung Jo ${ }^{1}$, Duck-Hye Lee ${ }^{2}$, Hyun-Ho Sung ${ }^{1}$ \\ ${ }^{1}$ Department of Clinical Laboratory Science, Dongnam Health University, Suwon, Korea \\ ${ }^{2}$ Department of Dental Technology, Dongnam Health University, Suwon, Korea
}

\section{한국 임상병리사들의 은퇴 기대 조사}

\author{
조윤경 ${ }^{1}$, 이덕혜 $^{2}$, 성현호 $^{1}$ \\ ${ }^{1}$ 동남보건대학교 임상병리과, ${ }^{2}$ 동남보건대학교 치기공과
}

\begin{abstract}
This study investigated the influence factors of retirement expectations among medical technologists at medical centers in Seoul and Gyeonggi area, Korea. Subjects were 158 medical technologists. Using a sampling survey method, data was analyzed between July 2015 and December 2016. There was a negative correlation between educational level and economic preparation, but gender and monthly income did not reach retirement expectation level. Conversely, we found no change in the trends with respect to gender, monthly income; nonetheless, we observed that frustration gradually increased with age and working duration. In conclusion, our findings show that there are four factors that significantly influence retirement expectation and preparation: age, level of education, working duration, and family condition. This study contributes to understanding retirement expectations and can be a reference for retirement preparation in medical technologists.
\end{abstract}

Key words: Medical Technologist, Retirement expectation, Retirement preparation

This is an Open Access article distributed under the terms of the Creative Commons Attribution Non-Commercial License (http://creativecommons.org/licenses/by-nc/4.0) which permits unrestricted non-commercial use, distribution, and reproduction in any medium, provided the original work is properly cited.

Copyright @ 2017 The Korean Society for Clinical Laboratory Science. All rights reserved.
Corresponding author: Hyun-Ho Sung

Department of Clinical Laboratory Science,

Dongnam Health University, Suwon 16328 Korea

Tel: 82-31-249-6414

Fax: 82-31-249-6410

E-mail:wantyou7@dongnam.ac.kr

Received: May 1, 2017

Revised $1^{\text {st. }}$ : May 28, 2017

Revised 2 ${ }^{\text {nd: }}$ June 4, 2017

Revised 3 ${ }^{\text {th }}$ June 8, 2017

Revised $4^{\text {th }}$ : June 8, 2017

Accepted: June 9, 2017

\section{INTRODUCTION}

Retirement is withdrawal from one's occupation or position, where a person stops employment especially upon reaching a certain age. In the Korea, while the normal retirement age historically has been age 55 , it was changed to age 60 from 2016. In most developed countries, populations are aging rapidly due to long-term declines of fertility and increasing longevity. With 65-year-old population currently reached $14.46 \%$ and the number of older Koreans expected to nearly double by 2060 [1]. These demographic developments have important implications for public finances, pension systems, labor markets, and organizations. Extending the working lives of older individuals are often perceived as a key policy response to population aging, putting the issue of retirement high on the policy agenda as well as the scientific agenda [2]. While the popular press has primarily focused on the factors associated with general retiree happiness, the academic literature has concentrated more specifi- 
cally on concepts related to psychological well-being. A review of this academic literature indicated that various definitions of psychological well-being have been utilized.

Comprising Medical Technologists, the laboratory medicine workforce has a vital role in the health care system, managing and applying evidence-based, scientific testing techniques to support patient care and protect against public health threats. In 2014, there were an estimated 49,936 Medical Technologists in the Korea, including 20,500 in community practice, nearly $65.9 \%$ of this workforce is female [3].

Medical Technologists are responsible for collecting, processing, and testing body fluids, thus providing physicians with critical information used in patient diagnosis, treatment, and monitoring of disease states. In the Korea, there are two main pathways for entry into practice at the bench technician level: the three-year associate degree and the four-year baccalaureate degree. Despite the recent Korea economic downturn, employment opportunities in the medical laboratory field continue to grow as shown in 2014, more than 20,500 medical laboratory professionals were employed in clinic and hospital laboratories with a projected increase of 30\% by 2024 [4]. An aging population requiring increased medical care, advancements in testing methodologies and an increased retirement rate of practicing laboratory professionals, has resulted in a shortage of Medical Technologists the Korea.

The one thing on which essentially all retirement scholars agree is that there is no generally accepted definition of the term "retirement." Hence, it is not surprising that a plethora of competing models of the stages of retirement has been generated [5]. Retirement expectation is an assessment of what life will be like after retirement. However, few studies have considered these factors in relation to retirement expectation, and many of these have focused on specific outcomes or occupations.

This study provides specific data array of factors, household and individual level financial, and retirement expectation characteristics for Medical Technologists, and influence retirement plans.

\section{MATERIALS AND METHODS}

\section{Sample and setting}

This study was part of a retirement expectation survey of Medical Technologist's attitudes of retirement preparations at Seoul and Gyeonggi area in the Korea. In this paper, by using the sampling survey data were completed from July 2015 to December 2016. A total of 37 participants provided the data for the retirement surveys. Ninety-two percent of the subjects were male and 8 percent were female. Eight percent of the subjects had less than a high school degree; 77 percent had a high school degree; 15 percent had a college degree or higher. All participants volunteered for the study, and all were assured that their responses were confidential. The collected register data were anonymized and de-identified prior to the analyses. Average age of the participants was 60.9 at male and 61.5 at female, as presented in Table 1.

Table 1. General characteristics of subjects (missing value exclude)

\begin{tabular}{llc}
\hline \multicolumn{1}{c}{ Characteristics } & Classifications & N (\%) \\
\hline Gender & Male & $63(39.9)$ \\
& Female & $94(60.1)$ \\
& Total & $157(100.0)$ \\
Age (years) & $20 \sim 29$ & $38(24.4)$ \\
& $30 \sim 39$ & $55(35.3)$ \\
& $40 \sim 49$ & $52(33.3)$ \\
& $\geq 50$ & $11(7.1)$ \\
Educational level & Total & $156(100.0)$ \\
& College & $56(35.4)$ \\
& University & $81(51.3)$ \\
& Master's & $19(12.0)$ \\
& Doctor's & $2(1.3)$ \\
Working period (years) & Total & $158(100.0)$ \\
& $\leq 5$ & $34(21.5)$ \\
& $6 \sim 10$ & $37(23.4)$ \\
& $11 \sim 15$ & $28(17.7)$ \\
& $16 \sim 20$ & $37(23.4)$ \\
& $\geq 21$ & $22(13.9)$ \\
& Total & $158(100.0)$ \\
& Married & $94(60.3)$ \\
Marital status & Single & $62(39.7)$ \\
& total & $156(100)$ \\
Monthly income (thousands) & $\leq 200$ & $5(4.6)$ \\
& $201 \sim 300$ & $17(15.6)$ \\
& $301 \sim 400$ & $16(14.7)$ \\
& & $15(13.8)$ \\
& & $56(51.4)$ \\
& & $109(100.0)$ \\
\hline & &
\end{tabular}




\section{Retirement expectation scale}

The questionnaire for the study was designed by Bae \& Jeon, in precedent research on "The effect of variable on attitudes and preparations toward retirement" [6]. Following procedures used in our previous research, 4 broad subdomains of retirement conditions were examined, namely general characteristics 6 questions, personal variables 2 questions, retirement expectations 20 questions and retirement preparations plan 14 questions.

The personal variables, retirement expectations and retirement preparations plan were Likert 5 point scales: The scores ranged from one point (not at all) to five points (very much). Individual variables were health and self esteem questions. The higher the score, the higher the health and self - esteem.

The retirement expectations (20 questions) were: The frustration included 6 questions (high scores indicate high frustration), which all loaded on reliability (0.91), meaning of rest (0.67), meaning of continuous life (0.71), The retirement preparations (14 questions) were: physical preparation (0.70), psychological preparation (0.61), and economic preparation (0.85). Cronbach's alpha is a statistic used as a measure of questionnaires all item.

\section{Statistical analysis}

Statistical analysis was performed using the SPSS, PC, Version 21.0 (SPSS, Chicago, USA) program. Differences in baseline characteristics between general characteristics and personal variables were tested for significance using the t-test, ANOVA or chi-square test. In addition, the multiple regression analysis was performed according to the correlation. All statistical significance level was presented as proportions and mean with 95\% confidence intervals (set as $p<0.05$ ).

\section{RESULTS}

\section{General characteristics of subjects}

A total of 158 (60.1\% females, 39.9\% males) working on the Medical Facilities of the Korea participated in this study. In this study, the distribution to age was 38 (24.4\%) of the $20 \mathrm{~s}, 55(22.5 \%)$ of the $30 \mathrm{~s}, 52(33.3 \%)$ of the $40 \mathrm{~s}$, $11(7.1 \%)$ the over 50 years old. The participants' educational level distribution was $56(35.4 \%)$ of college, 81 (51.3\%) of university, 19 (12.0\%) of master's, and the Graduated they to do Doctor's was 2 (1.3\%). The educational level was found to be the highest, especially for university. The working period of the subjects was 34 (21.5\%) said less than 5 years, 37 (23.4\%) said 6 to 10 years, $28(17.7 \%)$ said 11 to 15 years, $37(23.4 \%)$ said 16 to 20 years and 22 (13.9\%) said over 21 years. The participants' married and single the distributions were 94 (60.3\%) and $62(39.7 \%)$ of respectively. The monthly income levels were found to be the highest in the over 5 million won. General characteristic of the participants was presented in Table 1.

\section{Differences by retirement expectation according to general characteristics}

Retirement expectation analysis according to sex showed that was no statistical difference between male and female. We split the 4 groups by age and analyzed retirement expectation. Regarding frustration and rest in opinion around retirement was larger in 20s than 30s, were significant $(p<0.05)$. However, other characteristics were not statistically different (Table 2 ).

\section{Differences by retirement preparation according to general characteristics}

For both retirement psychological preparation and economic preparation these group were statistically different between age groups, educational level, and working period. Means, standard deviations between measures are presented in Table 3.

\section{Correlation of retirement expectation, preparation and general characteristics}

As shown in Table 4, presents the Pearson correlations for variables. Age was positive correlated with frustration $(\mathrm{r}=0.16, p<0.05)$ and economic $(\mathrm{r}=0.27, p<0.05)$. Age was negative correlated with rest $(\mathrm{r}=-0.17, p<0.05)$ and 
Table 2. Differences by retirement expectation according to general characteristics

\begin{tabular}{|c|c|c|c|c|c|c|}
\hline \multirow{2}{*}{ Characteristics } & \multirow{2}{*}{ Classifications } & Frustration & Hope & Rest & Continuous life & R-expectation \\
\hline & & $M \pm S D$ & $M \pm S D$ & $\mathrm{M} \pm \mathrm{SD}$ & $M \pm S D$ & $M \pm S D$ \\
\hline \multirow[t]{3}{*}{ Gender $^{\dagger}$} & Male & $2.32 \pm 0.71$ & $3.44 \pm 0.49$ & $2.61 \pm 0.83$ & $2.79 \pm 0.57$ & $3.57 \pm 0.59$ \\
\hline & Female & $2.15 \pm 0.70$ & $3.45 \pm 0.49$ & $2.79 \pm 0.91$ & $2.80 \pm 0.74$ & $3.59 \pm 0.59$ \\
\hline & $\mathrm{F} / \mathrm{t}$ & $0.57 / 1.41$ & $0.02 /-0.11$ & $0.68 /-1.27$ & $7.48 * /-0.05$ & $0.02 /-0.31$ \\
\hline \multirow[t]{5}{*}{ Age $^{\ddagger}$ (years) } & $20 \sim 29^{a}$ & $1.95 \pm 0.58$ & $3.51 \pm 0.48$ & $3.14 \pm 0.81$ & $2.68 \pm 0.63$ & $3.68 \pm 0.58$ \\
\hline & $30 \sim 39^{b}$ & $2.34 \pm 0.69$ & $3.43 \pm 0.50$ & $2.52 \pm 0.77$ & $2.81 \pm 0.62$ & $3.55 \pm 0.59$ \\
\hline & $40 \sim 49^{c}$ & $2.20 \pm 0.77$ & $2.20 \pm 0.77$ & $2.68 \pm 0.93$ & $2.68 \pm 0.93$ & $3.60 \pm 0.59$ \\
\hline & $\geq 50^{d}$ & $2.56 \pm 0.63$ & $2.56 \pm 0.63$ & $2.60 \pm 0.92$ & $2.60 \pm 0.65$ & $3.46 \pm 0.64$ \\
\hline & $\mathrm{F} /(\mathrm{a}, \mathrm{b}, \mathrm{c}, \mathrm{d})$ & $3.21 * /(a>b) *(c, d)$ & $0.39 /(a, b, c, d)$ & $4.06^{*} /(a>b) *(c, d)$ & $0.74 /(a, b, c, d)$ & $0.54 /(a, b, c, d)$ \\
\hline \multirow[t]{5}{*}{ Educational level $^{\ddagger}$} & College $\mathrm{a}^{\mathrm{a}}$ & $2.19 \pm 0.64$ & $3.37 \pm 0.54$ & $2.66 \pm 0.90$ & $2.85 \pm 0.73$ & $3.45 \pm 0.72$ \\
\hline & University $^{\mathrm{b}}$ & $2.25 \pm 0.72$ & $3.50 \pm 0.48$ & $2.69 \pm 0.87$ & $2.75 \pm 0.65$ & $3.65 \pm 0.54$ \\
\hline & Master's $s^{c}$ & $2.13 \pm 0.82$ & $3.52 \pm 0.32$ & $3.00 \pm 0.73$ & $2.91 \pm 0.63$ & $3.73 \pm 0.46$ \\
\hline & Doctor's ${ }^{d}$ & $2.75 \pm 1.06$ & $3.08 \pm 0.58$ & $2.83 \pm 2.12$ & $2.33 \pm 0.47$ & $3.25 \pm 0.82$ \\
\hline & $F /(a, b, c, d)$ & $0.57 /(a, b, c, d)$ & $1.26 /(a, b, c, d)$ & $0.74 /(a, b, c, d)$ & $0.71 /(a, b, c, d)$ & $1.85 /(a, b, c, d)$ \\
\hline \multirow{6}{*}{$\begin{array}{l}\text { Working period } \\
\text { (years) }\end{array}$} & $\leq 5^{a}$ & $1.95 \pm 0.60$ & $3.50 \pm 0.47$ & $3.09 \pm 0.72$ & $2.65 \pm 0.55$ & $3.66 \pm 0.57$ \\
\hline & $6 \sim 10^{b}$ & $2.22 \pm 0.71$ & $3.50 \pm 0.47$ & $2.63 \pm 0.94$ & $2.86 \pm 0.73$ & $3.63 \pm 0.61$ \\
\hline & $11 \sim 15^{c}$ & $2.36 \pm 0.72$ & $3.30 \pm 0.49$ & $2.53 \pm 0.79$ & $2.78 \pm 0.66$ & $3.39 \pm 0.52$ \\
\hline & $16 \sim 20^{d}$ & $2.22 \pm 0.66$ & $3.40 \pm 0.44$ & $2.74 \pm 0.88$ & $2.91 \pm 0.66$ & $3.54 \pm 0.53$ \\
\hline & $\geq 21^{\mathrm{e}}$ & $2.43 \pm 0.83$ & $3.54 \pm 0.61$ & $2.50 \pm 0.99$ & $2.77 \pm 0.81$ & $3.69 \pm 0.72$ \\
\hline & $F /(a, b, c, d, e)$ & $1.97 /(a, b, c, d, e)$ & $1.13 /(a, b, c, d, e)$ & $2.38 /(\mathrm{a}, \mathrm{b}, \mathrm{c}, \mathrm{d}, \mathrm{e})$ & $0.75 /(a, b, c, d, e)$ & $1.17 /(a, b, c, d, e$ \\
\hline \multirow[t]{3}{*}{ Marital status $^{\dagger}$} & Married & $2.19 \pm 0.76$ & $3.41 \pm 0.47$ & $2.88 \pm 0.84$ & $2.75 \pm 0.67$ & $3.54 \pm 0.58$ \\
\hline & Single & $2.24 \pm 0.67$ & $3.47 \pm 0.50$ & $2.62 \pm 0.90$ & $2.83 \pm 0.68$ & $3.61 \pm 0.60$ \\
\hline & $\mathrm{F} / \mathrm{t}$ & $0.09 /-0.63$ & $0.84 /-0.68$ & $0.96 / 1.80$ & $0.04 /-0.76$ & $0.01 /-0.72$ \\
\hline \multirow{6}{*}{$\begin{array}{l}\text { Monthly income } \\
\text { (thousands) }\end{array}$} & $\leq 200^{\mathrm{a}}$ & $2.20 \pm 0.43$ & $3.63 \pm 0.50$ & $2.93 \pm 0.86$ & $3.00 \pm 0.62$ & $3.60 \pm 0.58$ \\
\hline & $201 \sim 300^{b}$ & $2.20 \pm 0.83$ & $3.17 \pm 0.33$ & $2.82 \pm 0.98$ & $2.84 \pm 0.73$ & $3.30 \pm 0.48$ \\
\hline & $301 \sim 400^{c}$ & $2.57 \pm 0.76$ & $3.34 \pm 0.58$ & $2.66 \pm 0.79$ & $2.81 \pm 0.66$ & $3.40 \pm 0.73$ \\
\hline & $401 \sim 500^{d}$ & $2.35 \pm 0.81$ & $3.64 \pm 0.53$ & $2.48 \pm 0.91$ & $2.75 \pm 0.77$ & $3.84 \pm 0.61$ \\
\hline & $\geq 501^{\mathrm{e}}$ & $2.14 \pm 0.67$ & $3.46 \pm 0.49$ & $2.68 \pm 0.91$ & $2.88 \pm 0.72$ & $3.59 \pm 0.57$ \\
\hline & $F /(a, b, c, d, e)$ & $1.17 /(a, b, c, d$, & $23 /(a, b, c, d, e$ & $0.37 /(a, b, c, d, e$ & $0.15 /(a, b, c, d$, & $1.99 /(a, b, c, d, e$ \\
\hline
\end{tabular}

${ }^{*} p<0.05,{ }^{* *} p<0.01 .{ }^{\dagger} p$-values were calculated by t-test. ${ }^{\dagger} p$-values were calculated by one-way ANOVA.

Abbreviation: R-, Retirement.

psychological ( $\mathrm{r}=-0.21, p<0.01)$. Educational level was significantly correlated with economic and retirement preparation $(\mathrm{r}=0.17, p<0.05)$. There were increase relation in frustration $(\mathrm{r}=0.18, p<0.05)$ and economic preparation $(\mathrm{r}=0.28, p<0.01)$ in the working period. However, a decrease showed opinion of rest $(\mathrm{r}=-0.16, p<$ 0.05). The increase was statistically significant. Marital status was positive correlated only with economic $(p<$ 0.05).

\section{Multiple regression analysis of retirement expectation to general characteristics}

The multiple regression analyses were based on age group and working period, except for gender, education level, marital status, monthly income and for which frustration and rest were applied. Because gender, educa- tion level, marital status and monthly income were not significantly associated with hope or rest, continuous life, retirement expectation, they were not entered in the regression analysis. The analysis showed that age groups and working period were $\mathrm{R}^{2}$ value $2 \%$, affect to frustration. The increase in recognition of rest around retirement was larger in participants who were the younger and short working period. But the increase in frustration around retirement was larger in participants who were the older person, longer working period (Table 5).

\section{Multiple regression analysis of retirement preparation to general characteristics}

Age, educational level, working period, marital status Psychological, Economic and Preparation did physical activity were included in the multiple regression analysis. 
Table 3. Differences by retirement preparation according to general characteristics

\begin{tabular}{|c|c|c|c|c|c|}
\hline \multirow{2}{*}{ Characteristics } & \multirow{2}{*}{ Classifications } & Physical & Psychological & Economic & R-preparation \\
\hline & & $\mathrm{M} \pm \mathrm{SD}$ & $\mathrm{M} \pm \mathrm{SD}$ & $M \pm S D$ & $\mathrm{M} \pm \mathrm{SD}$ \\
\hline \multirow[t]{3}{*}{ Gender $^{\dagger}$} & Male & $3.17 \pm 0.64$ & $3.34 \pm 0.61$ & $2.90 \pm 0.76$ & $3.14 \pm 0.49$ \\
\hline & Female & $3.35 \pm 0.63$ & $3.40 \pm 0.65$ & $2.93 \pm 0.73$ & $3.22 \pm 0.47$ \\
\hline & $\mathrm{F} / \mathrm{t}$ & $0.01 /-1.71$ & $0.83 /-0.49$ & $0.68 /-0.25$ & $0.64 /-1.08$ \\
\hline \multirow[t]{5}{*}{$\operatorname{Age}^{\ddagger}$ (years) } & $20 \sim 29^{a}$ & $3.08 \pm 0.58$ & $3.64 \pm 0.55$ & $2.58 \pm 0.80$ & $3.09 \pm 0.45$ \\
\hline & $30 \sim 39^{b}$ & $3.29 \pm 0.62$ & $3.27 \pm 0.59$ & $2.94 \pm 0.58$ & $3.16 \pm 0.42$ \\
\hline & $40 \sim 49^{c}$ & $3.48 \pm 0.63$ & $3.45 \pm 0.65$ & $3.12 \pm 0.70$ & $3.35 \pm 0.51$ \\
\hline & $\geq 50^{d}$ & $3.00 \pm 0.76$ & $2.81 \pm 0.50$ & $3.14 \pm 1.00$ & $2.98 \pm 0.54$ \\
\hline & $\mathrm{F} /(\mathrm{a}, \mathrm{b}, \mathrm{c}, \mathrm{d})$ & $3.74^{*} /(a, b, c, d)$ & $6.45^{\star \star} /(a>b, d) *(c)$ & $4.52^{\star *} /(a, b, c, d)$ & $3.21^{*} /(a, b, c, d)$ \\
\hline \multirow[t]{5}{*}{ Educational level $^{\ddagger}$} & College $^{a}$ & $3.18 \pm 0.63$ & $3.30 \pm 0.59$ & $2.85 \pm 0.72$ & $3.11 \pm 0.48$ \\
\hline & University $^{\mathrm{b}}$ & $3.29 \pm 0.64$ & $3.44 \pm 0.68$ & $2.88 \pm 0.73$ & $3.20 \pm 0.48$ \\
\hline & Master's $s^{c}$ & $3.53 \pm 0.56$ & $3.36 \pm 0.51$ & $3.13 \pm 0.62$ & $3.34 \pm 0.42$ \\
\hline & Doctor's ${ }^{d}$ & $3.30 \pm 1.27$ & $3.00 \pm 0.47$ & $4.50 \pm 0.70$ & $3.60 \pm 0.34$ \\
\hline & $\mathrm{F} /(\mathrm{a}, \mathrm{b}, \mathrm{c}, \mathrm{d})$ & $1.47 /(a, b, c, d)$ & $0.71 /(a, b, c, d)$ & $3.99^{* *} /(d>a, c)^{*}(b)$ & $1.67 /(a, b, c, d)$ \\
\hline \multirow{6}{*}{$\begin{array}{l}\text { Working period }{ }^{\dagger} \\
\text { (years) }\end{array}$} & $\leq 5^{\mathrm{a}}$ & $3.06 \pm 0.62$ & $3.59 \pm 0.56$ & $2.58 \pm 0.81$ & $3.07 \pm 0.46$ \\
\hline & $6 \sim 10^{b}$ & $3.35 \pm 0.62$ & $3.39 \pm 0.52$ & $2.91 \pm 0.63$ & $3.22 \pm 0.40$ \\
\hline & $11 \sim 15^{c}$ & $3.25 \pm 0.48$ & $3.17 \pm 0.57$ & $2.84 \pm 0.56$ & $3.09 \pm 0.38$ \\
\hline & $16 \sim 20^{d}$ & $3.40 \pm 0.69$ & $3.36 \pm 0.69$ & $3.11 \pm 0.71$ & $3.29 \pm 0.52$ \\
\hline & $\geq 21^{e}$ & $3.34 \pm 0.75$ & $3.30 \pm 0.79$ & $3.24 \pm 0.84$ & $3.29 \pm 0.63$ \\
\hline & $\mathrm{F} /(\mathrm{a}, \mathrm{b}, \mathrm{c}, \mathrm{d}, \mathrm{e})$ & $1.51 /(a, b, c, d, e)$ & $1.84 /(a, b, c, d, e)$ & $3.78^{\star \star} /(d>a)^{\star}(b, c, e)$ & $1.51 /(\mathrm{a}, \mathrm{b}, \mathrm{c}, \mathrm{d}, \mathrm{e})$ \\
\hline \multirow[t]{3}{*}{ Marital status $^{\dagger}$} & Married & $3.30 \pm 0.62$ & $3.34 \pm 0.59$ & $3.00 \pm 0.65$ & $3.22 \pm 0.46$ \\
\hline & Single & $3.19 \pm 0.62$ & $3.43 \pm 0.67$ & $2.72 \pm 0.75$ & $3.11 \pm 0.47$ \\
\hline & $\mathrm{F} / \mathrm{t}$ & $1.42 / 1.33$ & $1.81 /-0.87$ & $2.05 / 2.45$ & $0.00 / 1.47^{\star}$ \\
\hline \multirow{6}{*}{$\begin{array}{l}\text { Monthly income } \\
\text { (thousands) }\end{array}$} & $\leq 200^{\mathrm{a}}$ & $3.48 \pm 0.70$ & $3.26 \pm 0.49$ & $2.84 \pm 1.39$ & $3.19 \pm 0.68$ \\
\hline & $201 \sim 300^{b}$ & $3.10 \pm 0.52$ & $3.54 \pm 0.66$ & $2.61 \pm 0.71$ & $3.08 \pm 0.41$ \\
\hline & $301 \sim 400^{c}$ & $3.02 \pm 0.66$ & $3.18 \pm 0.53$ & $3.13 \pm 0.79$ & $3.11 \pm 0.42$ \\
\hline & $401 \sim 500^{d}$ & $3.30 \pm 0.83$ & $3.42 \pm 0.91$ & $3.06 \pm 0.99$ & $3.26 \pm 0.73$ \\
\hline & $\geq 501^{\mathrm{e}}$ & $3.39 \pm 0.56$ & $3.32 \pm 0.68$ & $3.01 \pm 0.62$ & $3.24 \pm 0.48$ \\
\hline & $\mathrm{F} /(\mathrm{a}, \mathrm{b}, \mathrm{c}, \mathrm{d}, \mathrm{e})$ & $1.58 /(a, b, c, d, e)$ & $0.64 /(a, b, c, d, e)$ & $1.26 /(a, b, c, d, e)$ & $0.46 /(a, b, c, d, e)$ \\
\hline
\end{tabular}

${ }^{\star} p<0.05,{ }^{* *} p<0.01$.

Abbreviation: R-, Retirement.

Table 4. Correlation of retirement expectation, preparation and general characteristics

\begin{tabular}{|c|c|c|c|c|c|c|c|c|c|c|c|c|c|c|c|}
\hline Variable & A & B & C & $D$ & E & $F$ & G & $\mathrm{H}$ & I & $J$ & K & L & M & N & $\mathrm{O}$ \\
\hline$A$ & 1 & & & & & & & & & & & & & & \\
\hline B & -0.13 & 1 & & & & & & & & & & & & & \\
\hline C & 0.01 & $0.26^{\star \star}$ & 1 & & & & & & & & & & & & \\
\hline D & -0.07 & $0.88^{\star \star}$ & $0.20^{*}$ & 1 & & & & & & & & & & & \\
\hline E & 0.14 & $0.55^{\star \star}$ & 0.10 & $0.59^{\star \star}$ & 1 & & & & & & & & & & \\
\hline $\mathrm{F}$ & 0.10 & $0.43^{\star \star}$ & 0.03 & $0.48^{\star \star}$ & $0.67^{\star \star}$ & 1 & & & & & & & & & \\
\hline G & -0.11 & $0.16^{\star}$ & 0.29 & $0.18^{\star}$ & 0.03 & -0.08 & 1 & & & & & & & & \\
\hline $\mathrm{H}$ & 0.23 & -0.06 & 0.08 & -0.03 & 0.05 & 0.12 & $-0.34^{\star \star}$ & 1 & & & & & & & \\
\hline 1 & 0.09 & $-0.17^{\star}$ & 0.95 & $-0.16^{*}$ & -0.14 & -0.06 & 0.05 & $-0.22^{\star *}$ & 1 & & & & & & \\
\hline$J$ & 0.02 & 0.08 & -0.03 & 0.07 & 0.06 & 0.00 & 0.05 & 0.02 & -0.12 & 1 & & & & & \\
\hline K & 0.03 & -0.07 & 0.13 & -0.03 & 0.05 & 0.14 & $-0.43^{\star \star}$ & $0.93^{\star \star}$ & $-0.15^{\star}$ & $-0.16^{\star}$ & 1 & & & & \\
\hline$L$ & 0.14 & 0.13 & 0.15 & 0.14 & 0.15 & 0.15 & -0.11 & $0.19^{\star}$ & -0.07 & 0.07 & $0.17^{\star}$ & 1 & & & \\
\hline$M$ & 0.04 & $-0.21^{\star \star}$ & 0.03 & -0.14 & -0.09 & -0.04 & $-0.32^{* *}$ & 0.14 & 0.04 & -0.01 & $0.19^{*}$ & $0.25^{\star \star}$ & 1 & & \\
\hline$N$ & 0.02 & $0.27^{\star \star}$ & $0.17^{*}$ & $0.28^{\star \star}$ & $0.20^{\star \star \star}$ & 0.12 & 0.10 & 0.06 & -0.05 & 0.04 & 0.04 & $0.39^{\star *}$ & $0.16^{*}$ & 1 & \\
\hline $\mathrm{O}$ & 0.08 & 0.10 & $0.17^{\star}$ & 0.15 & 0.13 & 0.10 & -0.14 & $0.17^{*}$ & -0.04 & 0.05 & $0.18^{*}$ & $0.75^{\star \star}$ & $0.63^{\star \star}$ & $0.76^{\star \star}$ & 1 \\
\hline
\end{tabular}

${ }^{*} p<0.05,{ }^{* *} p<0.01$.

Abbreviation: A, Gender; B, Age; C, Educational level; D, Working period; E, Marital status; F, Monthly income; G, Frustration; H, Hope; I, Rest; J, Continuous life; K, Retirement expectation; L, Physical; M, Psychological; N, Economic; O, Retirement preparation. 
Table 5. Multiple regression analysis of retirement expectation to general characteristics

\begin{tabular}{|c|c|c|c|c|c|c|}
\hline $\begin{array}{l}\text { Retirement } \\
\text { expectation }\end{array}$ & Characteristics & $\beta$ & SE & $t$ & $D-W$ & $R / R^{2} / F$ \\
\hline \multirow[t]{2}{*}{ Frustration } & Age & 0.01 & 0.13 & 0.07 & \multirow[t]{2}{*}{2.01} & \multirow[t]{2}{*}{$1.84 / 0.02 / 2.66$} \\
\hline & Working period & 0.09 & 0.08 & 1.03 & & \\
\hline \multirow[t]{2}{*}{ Rest } & Age & -0.75 & 0.16 & -0.45 & \multirow[t]{2}{*}{1.84} & \multirow[t]{2}{*}{$0.17 / 0.03 / 2.45$} \\
\hline & Working period & -0.67 & 0.10 & -0.62 & & \\
\hline
\end{tabular}

Abbreviation: D-W, Durbin-Watson.

Table 6. Multiple regression analysis of retirement preparation to general characteristics

\begin{tabular}{|c|c|c|c|c|c|c|}
\hline $\begin{array}{l}\text { Retirement } \\
\text { preparation }\end{array}$ & Characteristics & $\beta$ & $S E$ & $t$ & $D-W$ & $\mathrm{R} / \mathrm{R}^{2} / \mathrm{F}$ \\
\hline Psychological & Age & -1.49 & 0.05 & $-2.70^{* *}$ & 2.00 & $1.84 / 0.02 / 7.32^{\star \star}$ \\
\hline \multirow[t]{4}{*}{ Economic } & Age & 0.05 & 0.13 & 0.38 & 2.13 & $0.30 / 0.09 / 3.79^{\star \star}$ \\
\hline & Educational level & 0.12 & 0.86 & 1.40 & & \\
\hline & Working period & 0.08 & 0.09 & 0.92 & & \\
\hline & Marital status & 0.09 & 0.14 & 0.63 & & \\
\hline Preparation & Educational level & 0.12 & 0.05 & $2.19^{*}$ & 2.20 & $0.17 / 0.03 / 4.79^{\star}$ \\
\hline
\end{tabular}

Abbreviation: D-W, Durbin-Watson.

With respect to retirement economic preparations toward general characteristics (age, educational level, working period, marital status), we found it to be affect significant. Age increase, that is, more negative psychological preparation about retirement, decreases psychological preparation (Table 6).

\section{DISCUSSION}

In this paper, we have presented the retirement expectation from Medical Technologists aged 20 through 60 years from several Seoul and Gyeonggi area, Korea, using data from the precedent questionnaire of retirement expectation survey and related them to general characteristics. Participants were participated in a study titled "The Investigation of the Retirement Expectation of Medical Technologists." After providing their informed consent, participants responded to the survey measures as indicated below. For all scales, unless stated otherwise, participants indicated their level of agreement with each item on scales ranging from 1 (not at all) to 5 (completely). Following completion of the survey, participants were debriefed and thanked for their involvement in the study

In industrialized countries increased life expectancy is pushing back retirement age as people realize that they might live decades in retirement [7]. In Korea, for example, where retirement is typically compulsory, people can expect to live 15 to 20 years after retirement from a primary job [8]. As a result, many older workers plan to work longer, postpone retirement, or return to the labor force after retirement.

We explore which Medical Technologists plan to retirement expectation and preparation after the statutory retirement age. Almost all employed late career workers plan to work until or beyond retirement age, especially men and those who have more educational and health resources, first, a higher education combined with a low job satisfaction predicts staying longer in the labor despite the wish to retire as early as possible. Second, individuals who have poor health and a low job satisfaction often wish to retire as early as possible but stay in the labor market until reaching to the compulsory retirement age. Thus, policy measures increasing merely the statutory retirement age create tensions, especially among those not satisfied with their jobs [9].

Physicians commonly reported retiring between 60 and 69 years of age. Excessive workload and burnout were frequently cited reasons for early retirement. Ongoing 
financial obligations delayed retirement, while strategies to mitigate career dissatisfaction, workplace frustration, and workload pressure supported continuing practice. Knowledge of when physicians plan to retire and how they can transition out of practice has been shown to aid succession planning [10]. With marital status as one of the most significant predictors of retirement wealth, planning for retirement income outside a married or partnered context is a determinant of wealth differences between single and married women [11]. Another 3 in 10 workers say that they worry about their personal finances while at work (30 percent). These stressed workers feel less financially secure and are far less confident about having enough money for a comfortable retirement than those who do not feel stressed. More than half of these workers believe they would be more productive at work if they didn't spend time worrying. Among all workers, about half say that retirement planning (53 percent), financial planning (49 percent), or health care planning ( 47 percent) programs would be helpful in increasing their productivity at work [12]. Transition into retirement should receive more attention from a nursing perspective since experiences such as, for example, grief, disconnectedness and loss of identity if unaddressed could lead to an unhealthy transition [13].

Retirement is often perceived to be an important life stage or event that requires significant planning and preparation [14]. In the Investigation of the retirement expectation of Medical Technologists based in Korea, retirement preparation was associated with a general characteristic, in both age and working period and with a smaller but decrease in the economic preparation of low educational level. In contrast, we found no change in the trends for gender, monthly income in relation to retirement, although, the cumulative of these frustration gradually increased with age and working period. These findings suggest that educational level and marital status affects retirement preparation but does not directly affect the risk of retirement expectation. These findings underscore the importance of studying retirement toward major life transitions like retirement. We contributed to the psychosocial approach to aging by showing that beyond positive aging, positive educational attitudes and retirement expectation toward age and working period are also protective against retirement frustration. In essence, the retirement expectation provides four factors to explain our findings: (a) retirement are likely part of the many concepts that we take in at a young age; (b) the Importance of Lifelong Education; (c) gain salience from working period; (d) relationship between marriage and retirement to impact retirement preparation. Construed based on general characteristics, we also show that retirement expectation can be extended to explain how preparation influence retirement. It can be concluded that adjustment to retirement was affected by the retirees' gender, marital status, level of education, type of job before retirement, job condition, and place of work. In addition, adequate resources as physical, financial, social support and mental capacity are associated with better adjustment to retirement.

Late in the past decade, the Great Recession hit the economy, a period of drastic decline in economic activity that led to large increases in unemployment rates and unemployment spell durations for workers of all ages. Older workers considering retirement in the last few years have faced a tough economic situation since the Great Recession, with a stubbornly sluggish recovery. Many older workers experienced unemployment and soaring duration of unemployment. Job loss, compensation reductions, declining home values, investment losses, and high debt have undermined retirement plans and expectations. Retirement is one of the most important transitions in a person's life, which also might affect health and well-being. In the present investigation, a comparison is made between the retirement goals of working Indian adults and previously published data on the retirement goals of working adults in the United States. Participants were 158 Indian respondents between 21 and 60 years of age. Each respondent completed a questionnaire in which they reported the nature of the goals they held for retirement. For the most part, the types of the goals enumerated by workers from India were similar to those of 
Americans. However, Indians were found to focus more on financial stability and self-related goals, whereas Americans tended to focus on leisure and exploration activities [15]. The correspondence between intentions and behavior varies primarily by health, education and type of work. Older workers with poor health, and workers with low education, often retire earlier than they prefer. Blue-collar workers often retire earlier than they had decided [16].

Therefore, in this analysis showed that age, level of education, family, the nature of the unit, and working conditions for retirement expectation affect significantly, the conclusion of this paper is helpful to understand the retirement expectation, to provide the certain reference to carry out retirement preparation. In china results show that age, level of the education, health, dependency ratio of the population, people of independent source of income, unit properties, working conditions will affect significantly for both men and women, but there is another bigger difference. The level of leisure consumption and the expected retirement age have a great effect on the retirement age relatively consistent [17].

Retirement is an important life course event that marks the start of a new life stage in which work is no longer dominant. Thus, employees have to adjust to the significant life changes that accompany the transition and seek to achieve psychological comfort with their retirement life [18]. For example, healthcare organizations might consider promoting retirement mentorship programs, resource toolkits, education sessions, and guidance around financial planning for physicians throughout their careers, as well as creating post-retirement opportunities that maintain institutional ties through teaching, mentoring, and peer support.

\section{요 약}

본 연구는 서울경기지역의 의료기관에 근무하는 임상병리사 158 명을 대상으로 은퇴기대에 영향을 미치는 요인을 찾고자 자 기기입식 설문조사를 실시하였다. 학력수준과 경제적 준비는 음의 상관성을 보였으나 성별, 월 소득 수준은 은퇴기대수준에
미치지 않는 요인이었다. 좌절감 수준에서는 연령과 근로시간 면에서 유의한 증가를 보였으며, 교육수준과 결혼 상태는 은퇴 준비에 미치는 요인이나 은퇴 기대에는 연관성이 없음을 알 수 있었다. 따라서, 연령, 교육수준, 가족, 은퇴기대 조건이 상호 연 관성을 미치는 요인임을 알 수 있었다. 결론적으로 본 연구는 의 료현장의 임상병리사들이 향후 은퇴기대 및 은퇴준비를 위해 영향인자를 발굴하는 기초정보를 제공하였고 향후 은퇴를 위한 교육, 심리적 수준, 직업특성을 고려하는 연구가 추가되어야할 것으로 사료된다.

\section{Acknowledgements: None}

Funding: This study was financially supported by Dongnam Health University.

Conflict of interest: None

\section{REFERENCES}

1. Statistics Korea. Estimated future population [Internet]. Daejeon: Statistics Korea; 2012 [cited 2016 Mar 2]. Available from: http://kosis.kr/statHtml/statHtml.do?orgId=101\&tblid=DT_ 1B35001\& vw_cd=MT_ZTITLE\&list_id=A41_10_10\&scrId=\&seq No=\&lang_mode=ko\&obj_var_id=\&itm_id=\&conn_path=E1\&path.

2. Marleen D. Understanding retirement processes: The role of life histories. Palgrave Macmillan, UK: Springer; 2017. p263-291.

3. Korea Research Institute for Vocational Education. 2014 Tomorrow's world of work. Seoul: Ministry of Education; 2014. p316-317.

4. Bureau of Labor Statistics. 2015 Employment projections program [Internet]. Washington: US Department of Labor; 2016 [cited 2017Mar 2]. Available from: https://www.bls.gov/news.release/pdf/ecopro.pdf.

5. Henderson DB. Reconceptualizing retirement: A status-based approach. J Aging Stud. 2016;38:1-5.

6. Bae MJ, Jeon KY. The effect of variable on attitudes and preparations toward retirement. J Korean Home Economics Association. 2004;42(7):89-102.

7. Kanfer R, Beier ME, Ackerman PL. Goals and motivation related to work in later adulthood: An organizing framework. European Journal of Work and Organizational Psychology. 2013;(22):253264. doi: http://doi.org/10.1080/1359432X.2012.734298.

8. Choi YO. 2016 Longevity risk in Korea. KDI Focus. Sejong: Korea Development Institute; 2016 August.

9. Lindemann K, Marge U. Trapped in 'involuntary' work in the late career? Retirement expectations versus the desire to retire in Estonia. Studies of Transition States and Societies. 2016;8(6): 60-77.

10. Glass JR, Kilpatrick BB. Gender comparisons of baby boomers and financial preparation for retirement. Educ Gerontol. 1998;24(8):719-745.

11. Butrica BA, Smith KE, Lams H. It's all relative: Understanding 
the retirement prospects of baby boomers. Research report. Chestnut Hill: Center for Retirement Research, Boston College; 2003 November. Research No. 2003-21.

12. Greenwald L, Copeland C, et al. The 2017 retirement confidence survey: Many workers lack retirement confidence and feel stressed about retirement preparations [Internet]. Washington: Employee Benefit Research Institute; 2017 [cited 2017 Mar 21]. Available from: https://www.ebri.org/publications/ib/index.cfm?fa=ibDisp\&content_id=3426.

13. Djukanović I, Peterson U. Experiences of the transition into retirement: An interview study. Nord J Nurs Res. 2016:36(4); 224-232.

14. Kopanidis F, Robinson L, et al. "'I'm not old enough!" Why older single women are not engaging in retirement planning services.
In: Petruzzellis L, Winer RS. editors. Rediscovering the essentiality of marketing. Cham: Springer International Publishing; 2016. p871-876.

15. Gupta R, Hershey DA. Cross-national differences in goals for retirement: the case of India and the United States. J Cross Cult Gerontol. 2016;31(3):221-236.

16. Solem PE, Syse A, Furunes T, Mykletun RJ, de Lange A, Schaufell $\mathrm{W}$, et al. To leave or not to leave: retirement intentions and retirement behavior. Ageing Soc. 2016;36(2):259-281.

17. Gong X. Research on gender differences of labor retired intend in China. Cross-Cultural Communication. 2016;12(8):66-70.

18. van Solinge H, Henkens K. Adjustment to and satisfaction with retirement: two of a kind? Psychol Aging. 2008;23(2):422-434. doi: 10.1037/0882-7974.23.2.422. 